\title{
Gait Planning and Sensory Feedback Control for Robotic Sensor Systems in Smart Cities
}

\author{
Helin Wang, Hao Zhang, Zhuping Wang, and Qijun Chen* \\ Department of Control Science and Engineering, Tongji University \\ No 4800 Cao'an Road, Jiading District, Shanghai 201804, China
}

(Received December 29, 2018; accepted March 11, 2019)

Keywords: smart cities, robotic sensor systems, sensory feedback control, gait planning

Robotic sensor systems play a vital role in the intelligent working of service robots, and service robots are important components of smart cities. Robotic sensors sense information from themselves and their environment, and fuse pieces of information for use in perception, decision, planning, and control. In this paper, we investigate the application of robotic sensor systems in 3D biped walking. By using sensory feedback control, offline gait planning and online gait modification schemes have been proposed. The gait planner plans the frontal and lateral reference trajectory of a biped robot. Moreover, the kinetic constraint, zero moment point (ZMP) stability criterion, and unilateral constraint are taken into account, simultaneously. The gait modification consists of the torso posture controller, the ZMP compensator, and the joint angle controller. It is utilized to reduce the robot joint error through posture control and ZMP compensation. Additionally, the online sensor parameter optimization based on reinforcement learning (RL) is proposed to improve the stable margin of walking. Finally, simulations are presented to illustrate the effectiveness of the proposed method.

\section{Introduction}

A smart city is an integration of sensory, control, and intelligent technologies. ${ }^{(1)}$ It is an urban area that makes use of different types of sensor to collect data information and manage equipment and resources efficiently. ${ }^{(2)}$ It is referred to as a frontier concept and exploratory practice in the process of promoting industrial and urban informatization. Its large-scale applications will become one of the new economic growth points in the future. ${ }^{(3)}$ The construction of a smart city will create a great demand for industry and will help people live conveniently. It is becoming a more effective and vital approach to make developed countries competitive in the long term. In 2004, Japan and Korea formulated the national strategic planning of U-Japan and U-Korea. America has focused on strengthening the construction of intelligent infrastructure and promoting smart city application projects. According to the statistics of the Chinese smart cities forum, 6 provinces and 51 cities have included smart cities in their government work reports in China. ${ }^{(4,5)}$

*Corresponding author: e-mail: wh17228@tongji.edu.cn

https://doi.org/10.18494/SAM.2019.2251 
Since sensors and their applications play a vital role in smart cities, it is significant to apply them to benefit humankind. How to sense, integrate, and fuse information from sensors is a core problem. Sensor perception acquires data knowledge from different sensors, which is the primary link of automatic detection and control. ${ }^{(6,7)}$ According to different sensing functions, sensors can be classified into, for example, thermal, force, magnetic, and gas sensors. Sensor integration combines sensory data or data derived from disparate sources, ${ }^{(8,9)}$ and sensor fusion fuses different pieces of sensing information into one representation. These sensor technologies have the advantages of diverse environmental information, high robustness, and less uncertainty. The branches of sensor integration and fusion include the integration pattern, the control structure, and the fusion algorithm. Currently, common integration patterns are neural networks and logical sensors. ${ }^{(10,11)}$ The former pattern focuses on the mapping relationship between the input and the output, and the latter pattern regards physical sensors as logical sensors. The control structure is selected according to different application scenarios. For example, distributed blackboard control is suitable in real-time situations, and adaptive learning has a better control effect in a dynamic environment. The essence of sensor fusion lies in the processing of uncertain information. Common fusion algorithms ${ }^{(12,13)}$ refer to, for example, the weighted mean, Kalman filter, multi-Bayesian estimation, and fuzzy logic. The weighted mean processes a set of redundant raw sensing data by weighted averaging. The Kalman filter has better real-time performance, which is usually utilized to deal with dynamic, low-level, and redundant data. The multi-Bayesian estimation regards all sensors as a group consisting of multiple decision makers. The fuzzy logic is a multivalued logic method, which represents uncertain sensor information in logical reasoning. One of the most advanced and challenging sensor application fields involves robotic sensor systems. Recent years have witnessed an increasingly rapid research on sensory feedback control for robotic sensor systems in smart cities. Numerous results have been obtained. ${ }^{(14,15)}$ Different sensors are utilized to make a robot accomplish humanlike movements. The rotating potentiometer detects robot joint rotation, and the six-dimensional force sensor reflects ground reaction force (GRF) information. Note that these works focus on the working principle and control methods of robotic sensors. However, there still exist numerous unsolved scientific challenges, such as gait planning, trajectory tracking, and environmental adaptability.

In housekeeping service, disaster rescue, and medical rehabilitation, 3D biped walking is a humanlike walking mode, which is more approximate to the natural walking environment than 2D biped walking. However, adaptive 3D stable biped walking is difficult to achieve, because it needs to balance the frontal and lateral movements simultaneously. ${ }^{(16)} 3 \mathrm{D}$ biped walking has the characteristics of multiphase, hybrid nature of legged locomotion and unilateral constraints, which must be satisfied by forces and torques at the foot-ground interface. Additionally, the complexity of an environment will also lead to instability ${ }^{(17)}$ and trajectory tracking error ${ }^{(18,19)}$ in biped walking.

To overcome the above problems, a hierarchical structure based on multisensor information feedback has been receiving increasing attention. It has four layers, namely, the decision, coordination, execution, and perception layers. The decision layer makes decisions according to the robot state, walking command, and environmental information. It then interacts with 
a remote master workstation through a wireless network. The coordination layer coordinates commands between the execution and decision layers. The execution layer carries out the trajectory tracking of each joint. The perception layer senses environmental information and obtains the real-time walking state of the robot. ${ }^{(20,21)}$ It is the precondition of stable biped walking. It consists of sensors including photoelectric encoders in each joint, force and torque sensors on the ankle, and upper body accelerometers. The sensors can perceive information and deal with a 3D biped walker with model uncertainties and trajectory tracking errors. Although many advanced results on sensor applications in biped walking have been reported in the literature, ${ }^{(22-24)}$ there are still some challenging issues to be addressed, including sensory feedback control and sensor parameter optimization. All these observations motivate us in our current study.

In this paper, we concentrate on a hierarchical biped walking structure based on multisensor information feedback with walking stability constraints and real-time sensory parameter regulations. The main contributions can be summarized as follows.

(1) A novel gait generation control strategy is proposed to plan the frontal and lateral reference trajectories for the biped robot, which takes the kinetic constraint, zero moment point (ZMP) stability criterion, and unilateral constraint into account. The selection of gait parameters is formulated as a constrained nonlinear optimization problem, which is in the form of the moment quadratic function with continuous state switching conditions.

(2) A sensory feedback mechanism is utilized for trajectory tracking. It is phase-dependent and consists of the torso posture controller, ZMP compensator, and joint angle controller. The torso posture controller senses the robot posture information with the MEMS accelerometer and gyroscope. The ZMP compensator uses force-sensing resistors (FSRs) to satisfy the walking stability criterion. The joint angle controller measures the angle and angular velocity of each joint with joint sensors.

(3) The online sensor parameter optimization is proposed on the basis of reinforcement learning (RL). The sensory feedback control parameters are automatically adjusted in each walking cycle. With this online learning scheme, 3D biped walking will satisfy gait planning constraints, and feedback parameters are also modified.

\section{Dynamic Model}

A complete walking step cycle of a biped robot can be divided into the double-support phase (DSP) and single-support phase (SSP). The link dynamic model of the biped robot is depicted in Fig. 1. It consists of five links: a torso and two legs with revolute knees and terminated with footplates. The weights of the shank and thigh are $m_{1}$ and $m_{2}$, and their lengths are $L_{1}$ and $L_{2}$, respectively. The robot here has a total of 9 degrees of freedom (dof) of motion: four in each leg and one in the upper body. Several assumptions are made in advance.

Assumption 1: The impact between the swing leg and the ground is fully elastic, which satisfies the conservation of angular momentum.

Assumption 2: There is no up-spring and lateral shift between the leg and the ground when the impact happens. 


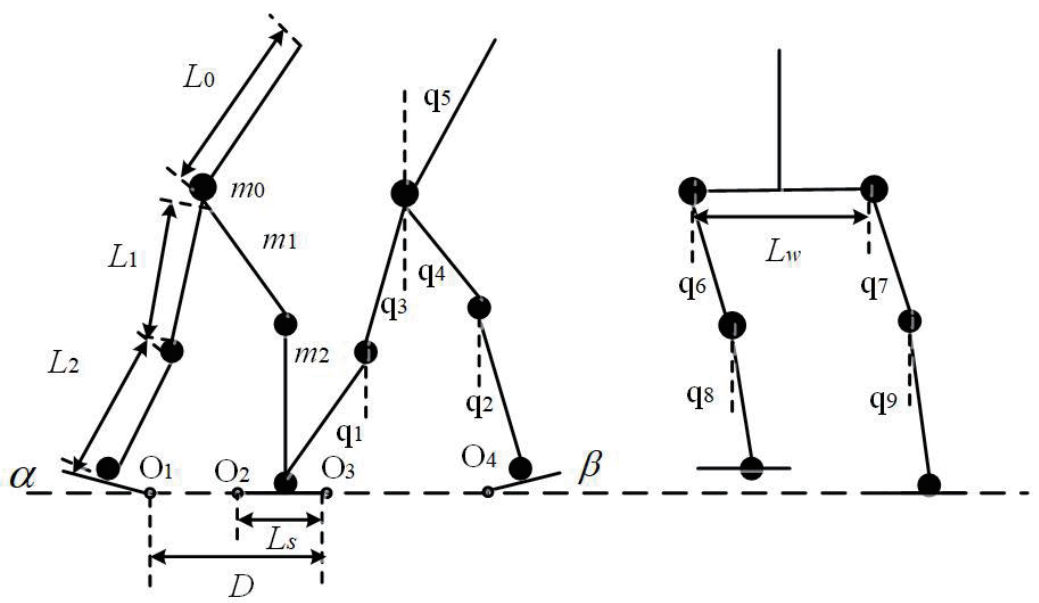

Fig. 1. Dynamic model.

Assumption 3: Because the impact is instantaneous, the angular velocity suddenly changes while its angular displacement remains unchanged at the impact.

To calculate the appropriate movement of the robot that leads to the given trajectory, the dynamics in the DSP is used, which can be derived from the Lagrange equation as

$$
\boldsymbol{M}(\boldsymbol{q}) \ddot{\boldsymbol{q}}+\boldsymbol{C}(\boldsymbol{q}, \dot{\boldsymbol{q}}) \dot{\boldsymbol{q}}+\boldsymbol{G}(\boldsymbol{q})=\boldsymbol{B} \boldsymbol{\tau}+\boldsymbol{J}^{\mathrm{T}} \boldsymbol{u}
$$

where $\boldsymbol{q}=\left[q_{1}, q_{2}, q_{3}, q_{4}, q_{5}, q_{5}, q_{6}, q_{7}, q_{8}, q_{9}\right]^{\mathrm{T}} \in \boldsymbol{R}^{9 \times 1}$ is the vector of generalized coordinates. $\boldsymbol{M}(\boldsymbol{q}) \in \boldsymbol{R}^{9 \times 9}$ is the symmetrical inertia matrix, which is positive definite, $\boldsymbol{C}(\boldsymbol{q}, \dot{\boldsymbol{q}}) \in \boldsymbol{R}^{9 \times 9}$ is the centrifugal force and Coriolis force matrix, $\boldsymbol{G}(\boldsymbol{q}) \in \boldsymbol{R}^{9 \times 1}$ is the gravity matrix, $\boldsymbol{B} \in \boldsymbol{R}^{9 \times 9}$ is the torque distribution matrix, $\tau \in \boldsymbol{R}^{9 \times 1}$ is the actuator torques matrix, $\boldsymbol{J}$ is the Jacobian matrix, $\boldsymbol{u}=\left[F_{\text {ext }}, \tau_{\text {ext }}\right]$ is composed of the GRF $F_{\text {ext }}$ and torque $\tau_{\text {ext }}$ in the DSP, and $\boldsymbol{u}=\mathbf{0}^{9 \times 1}$ in the SSP.

Remark 1: In this dynamic model, the coupling between the forward and backward directions have been taken into account, which precisely describes the biped robot, compared with the three-dimensional linear inverted pendulum (3D LIP). ${ }^{(25)}$

\section{Gait Planning}

To realize periodic stable walking, gait planning is carried out to discuss the coordination of each joint in a time sequence and task space. It is assumed that the walking movement takes place in the sagittal and lateral planes, and on a horizontal surface without obstacles. In this section, an offline gait planning method is introduced to control the bipedal to follow a stable reference trajectory in accordance with the ZMP stability criterion. 


\subsection{Kinematic constraints}

In this section, a series of kinematic constraints are discussed to complete gait pattern generation and realize continuous switching between the swing foot and the support foot, taking the joint angle and velocity into account. Each step should not be too low in order to improve the obstacle avoidance capability of biped robots. Moreover, there is point contact between the foot and the ground at the beginning and end of each step. Thus, it is necessary to divide every gait cycle into several subphases, including two parts in the SSP and three parts in the DSP.

In the initial stage of the SSP, the tiptoe leaves the ground and the swing foot is raised. The start time is assumed to be $t_{1}$ when the robot speed is 0 . The distance between the feet remains unchanged, and only the tip of the toe of the swing foot touches the ground. There exist the following constraints:

$$
\begin{gathered}
C_{s}^{1}=\boldsymbol{P}_{O_{2}}\left(\boldsymbol{q}\left(t_{1}\right)\right)-\boldsymbol{P}_{O_{1}}\left(\boldsymbol{q}\left(t_{1}\right)\right)=\left[\begin{array}{ll}
D-L_{s}-L_{w} & 0
\end{array}\right]^{\mathrm{T}}, \\
C_{s}^{2}=\boldsymbol{V}_{O_{2}}\left(\boldsymbol{q}\left(t_{1}\right), \dot{\boldsymbol{q}}\left(t_{1}\right)\right)=\frac{\partial \boldsymbol{P}_{O_{1}}\left(\boldsymbol{q}\left(t_{1}\right)\right)}{\partial \boldsymbol{q}} \dot{\boldsymbol{q}}\left(t_{1}\right)=\mathbf{0}^{3 \times 1}, \\
C_{s}^{3}=q_{2}\left(t_{1}\right)=-\alpha,
\end{gathered}
$$

where $\boldsymbol{P}_{O_{1}}$ and $\boldsymbol{P}_{O_{2}}$ are Cartesian coordinates of $O_{1}$ and $O_{2}$, and $D$ and $L_{s}$ are the step and foot lengths, respectively. $L_{w}$ is the distance between the two legs. $\alpha$ stands for the angle between the swing foot and the ground.

Then, in the middle stage of the SSP, the swing leg reaches the maximum height of the lifting step, where the footplate is parallel to the ground and the heel speed is 0 . The time is assumed to be $t_{1}$. Some kinetic constraints are described as

$$
\begin{gathered}
C_{s}^{4}=\boldsymbol{P}_{\text {foot }}^{\mathrm{T}}\left(\boldsymbol{q}\left(t_{2}\right)\right) \cdot \boldsymbol{e}_{z}=H_{m}, \\
C_{s}^{5}=\boldsymbol{V}_{\text {foot }}^{\mathrm{T}}\left(\boldsymbol{q}\left(t_{2}\right), \dot{\boldsymbol{q}}\left(t_{2}\right)\right) \cdot \boldsymbol{e}_{x}=\left(\frac{\partial \boldsymbol{P}_{\text {foot }}\left(\boldsymbol{q}\left(t_{2}\right)\right)}{\partial \boldsymbol{q}} \dot{\boldsymbol{q}}\left(t_{2}\right)\right)^{\mathrm{T}} \cdot \boldsymbol{e}_{x}=0,
\end{gathered}
$$

where $\boldsymbol{P}_{\text {foot }}$ and $\boldsymbol{V}_{\text {foot }}$ are the position and velocity at $t_{m}$, and $\boldsymbol{e}_{x}$ and $\boldsymbol{e}_{z}$ are unit direction vectors along the $x$ - and $z$-axes, respectively. $H_{m}$ is the step height.

At $t_{3}$, which is the end of the SSP as well as the start of the DSP, the swing foot completes a step action in air when the heel of the swing foot touches the ground. There are several kinetic constraints.

$$
C_{d}^{1}=\boldsymbol{P}_{O_{4}}\left(\boldsymbol{q}\left(t_{3}\right)\right)-\boldsymbol{P}_{O_{3}}\left(\boldsymbol{q}\left(t_{3}\right)\right)=\left[\begin{array}{lll}
D-L_{s} & L_{w} & 0
\end{array}\right]^{\mathrm{T}}
$$




$$
\begin{gathered}
C_{d}^{2}=\boldsymbol{V}_{O_{4}}\left(\boldsymbol{q}\left(t_{3}\right), \dot{\boldsymbol{q}}\left(t_{3}\right)\right)=\frac{\partial \boldsymbol{P}_{O_{4}}\left(\boldsymbol{q}\left(t_{3}\right)\right)}{\partial \boldsymbol{q}} \dot{\boldsymbol{q}}\left(t_{3}\right)=\mathbf{0}^{3 \times 1} \\
C_{d}^{3}=q_{2}\left(t_{3}\right)=\beta
\end{gathered}
$$

Here, $\beta$ refers to the angle between the heel and the ground at the foot placement.

In the middle stage of the DSP (at $t_{4}$ ), the foot is in full contact with the ground surface. At the end stage of the DSP (at $t_{5}$ ), the support foot begins to rotate around the toes. The constraints are

$$
\begin{aligned}
& C_{d}^{4}=q_{1}\left(t_{4}\right)=0, \\
& C_{d}^{5}=\dot{q}_{1}\left(t_{4}\right)=0, \\
& C_{d}^{6}=q_{2}\left(t_{5}\right)=0, \\
& C_{d}^{7}=\dot{q}_{2}\left(t_{5}\right)=0 .
\end{aligned}
$$

In accordance with continuous and periodic gait characteristics, the switch from the exchange of the swing foot to the support foot occurs at $t_{6}$.

$$
C_{w}\left\{\begin{array}{l}
q_{2}\left(t_{6}\right)=q_{1}\left(t_{1}\right), q_{4}\left(t_{6}\right)=q_{3}\left(t_{1}\right), q_{7}\left(t_{6}\right)=q_{6}\left(t_{1}\right), q_{9}\left(t_{6}\right)=q_{8}\left(t_{1}\right) \\
\dot{q}_{2}\left(t_{6}\right)=\dot{q}_{1}\left(t_{1}\right), \dot{q}_{4}\left(t_{6}\right)=\dot{q}_{3}\left(t_{1}\right), \dot{q}_{7}\left(t_{6}\right)=\dot{q}_{6}\left(t_{1}\right), \dot{q}_{9}\left(t_{6}\right)=\dot{q}_{8}\left(t_{1}\right)
\end{array}\right.
$$

Remark 2: By adding kinetic constraints for gait planning, previous walking phases including the SSP and dDSP are divided into several subphases. This is a clearer description of the 3D biped walking. These kinetic constraints are also the basis of the ZMP stability criterion and reinforce learning for parameter modification, which is discussed in detail in Sect. 4.

\subsection{ZMP stability criterion}

From the results of recent research, ${ }^{(25,26)}$ the most intuitive and popular choice for the ZMP position is set in the middle of the sole of the supporting foot, as shown in Fig. 2, where the tipping moments are zero. The ZMP was first put forward by Vukobratovic for measuring the dynamic stability conditions in biped walking. ${ }^{(27)}$ It can be defined as the point on the ground where the net moment of the inertial and gravity forces equals zero. $P$ stands for the ZMP in Fig. 2. In terms of stability, the robot is stable as long as there is a ZMP inside the supporting polygon. The polygon is the minimum domain of contact points between the foot and the ground. Thus, the ZMP position should be calculated to satisfy ZMP constraints. 


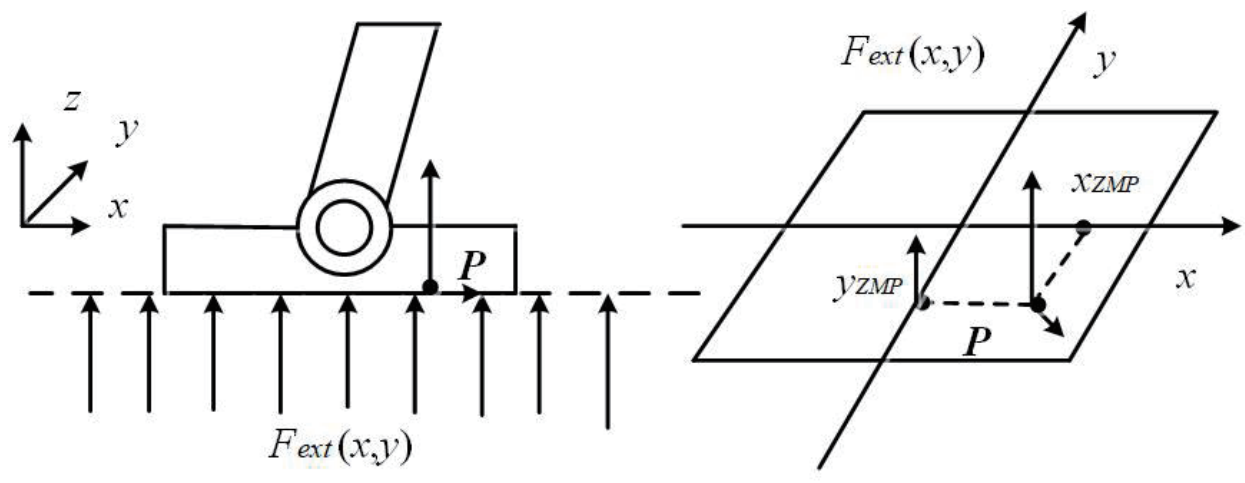

Fig. 2. Schematic diagram of ZMP.

The pressure distribution $F_{\text {ext }}(x, y)$ in Fig. 2 shows the reaction force from the ground. This force can be decomposed into $F_{\text {ext }}^{z}(x, y), F_{\text {ext }}^{x}(x, y)$, and $F_{\text {ext }}^{y}(x, y)$, where $F_{\text {ext }}^{z}(x, y)$ is the component perpendicular to the ground, and $F_{e x t}^{x}(x, y)$ and $F_{e x t}^{y}(x, y)$ are components tangential to the ground. The ZMP is defined as the point at which the moment generated by the reaction force satisfies $\tau_{e x t}^{x}=\tau_{e x t}^{y}=0$ on the ground. Let $\left(x_{z m p}, y_{z m p}\right)$ represent the coordinates of the ZMP on the foot,

$$
\begin{gathered}
\tau_{\text {ext }}^{x}=\iint_{S}\left(x-x_{z m p}\right) F_{e x t}^{z}(x, y) d x d y=0, \\
\tau_{\text {ext }}^{y}=\iint_{S}\left(y-y_{z m p}\right) F_{e x t}^{z}(x, y) d x d y=0,
\end{gathered}
$$

where the domain $S$ indicates the foot area and $\partial S$ represents a set consisting of all the boundary points in $S$. Thus, $\left(x_{z m p}, y_{z m p}\right)$ are calculated as

$$
\begin{aligned}
x_{z m p} & =\frac{\iint_{S} x F_{e x t}^{z}(x, y) d x d y}{F_{e x t}^{z}(x, y)}, \\
y_{z m p} & =\frac{\iint_{S} y F_{e x t}^{z}(x, y) d x d y}{F_{e x t}^{z}(x, y)} .
\end{aligned}
$$

Therefore, the ZMP constraints can be described as

$$
\sqrt{\left(x^{\prime}-x_{z m p}\right)^{2}+\left(y^{\prime}-y_{z m p}\right)^{2}} \geq \delta_{z m p}
$$

where $\left(x^{\prime}, y^{\prime}\right) \in \partial S, \delta_{z m p}$ is the ZMP stable threshold. 
On the other hand, some other constraints are also discussed to satisfy unilateral constraints. These constraints are aimed at satisfying the condition that the support foot touches the ground without slipping.

$$
\begin{gathered}
F_{\text {ext }}^{z}>0, \\
\sqrt{\left(F_{\text {ext }}^{x}\right)^{2}+\left(F_{\text {ext }}^{y}\right)^{2}}<\mu \cdot F_{\text {ext }}^{z},
\end{gathered}
$$

where $F_{e x t}^{x}, F_{e x t}^{y}$, and $F_{e x t}^{z}$ are the components of the GRF $F_{e x t}$ on the $x$-, $y$-, and $z$-axes of coordinates, respectively. $\mu$ is the friction coefficient.

\subsection{Walking parameter optimization}

On the basis of the above kinetic constraints [Eqs. (2)-(14)], ZMP constraint [Eq. (19)], and unilateral constraints [Eqs. (20) and (21)], the walking trajectory of each joint planning can be written in the form of piecewise fourth-order polynomials. By discretizing the continuous time, the $K$ th fourth-order polynomial is described as

$$
\left\{\begin{array}{l}
\Lambda_{t}=\left(t-t_{k}\right) /\left(t_{k+1}-t_{k}\right), \\
q_{i}(t)=\sum_{j=0}^{4} C_{i k j} \Lambda_{t}^{j}, t \in\left[\begin{array}{ll}
t_{k} & t_{k+1}
\end{array}\right]
\end{array} \quad(i=1,2,3, \ldots, 9),\right.
$$

where $\Lambda_{t}$ expresses the proportion of time in a walking cycle and $q_{i}(t)$ is the ith joint angular trajectory.

As for trajectory optimization, the torque minimization criterion is adopted to design the cost function $\boldsymbol{J}(\boldsymbol{q}, \dot{\boldsymbol{q}})$ :

$$
\boldsymbol{J}(\boldsymbol{q}, \dot{\boldsymbol{q}})=\int_{t_{1}}^{t_{3}} \tau^{\mathrm{T}}(t) \tau(t) d t+\int_{t_{3}}^{t_{6}}\left(\tau^{\mathrm{T}}(t) \tau(t)+\boldsymbol{u}^{\mathrm{T}}(t) \boldsymbol{u}(t)\right) d t .
$$

Equation (12) describes the quadratic integral of torque in the SSP and DSP. Therefore, gait planning becomes a classical nonlinear constraint optimization problem, which can be solved by sequential quadratic programming (SQP).

Remark 3: SQP is considered to be one of the most effective ways to solve nonlinear constrained optimization. It transforms the optimal trajectory and optimal control input into a nonlinear optimization problem with constraints. This method solves the local optimal solution of a stable periodic gait, which can be realized by the Fmincon function in the Matlab Optimization toolbox. Compared with other nonlinear optimization methods, SQP has a good convergence and boundary searching capability. 


\section{Sensory Feedback Control}

In spite of walking trajectory planning in advance, it is possible for the robot to fall while walking, owing to the differences between the dynamic model and the actual robot. Therefore, sensory feedback control must be utilized to realize automatic gait adjustment. Typical humanoid robots are equipped with many different types of sensor (e.g., gyroscope, acceleration sensor, FSRs in the feet, and sensors measuring the actual joint angles). In this section, sensor information regarding the torso and ankle is used to modify the preplanned trajectories, and the feedback control parameters are set by RL. The system block diagram with sensory feedback control is shown in Fig. 3.

Figure 3 shows the three main parts, namely, the torso posture controller, ZMP compensator, and joint angle controller. The first part is designed to prevent the deviation of the torso angle away from the ideal value. The middle part is aimed at reducing errors between actual and reference values. The last part acts as a feedback to convert the above control command to the actions of the robot.

\subsection{Posture controller}

The posture angle of the torso plays a vital role in stable robot walking. The torso posture sensors, including the accelerometer and rotating gyroscope, are easily affected by the walking process. The excessive tilt of the upper body will result in the center of mass (COM) deviating from the stable region. If an immediate recovery is not adopted, the robot will fall down. Thus, one of the effective methods of posture recovery is to adjust the hip joint angle of the support foot. With the information from the torque sensor installed on the foot, the robot decision layer can decide which leg is the support leg. The torso posture sensors installed in the upper body then modify the desired hip joint in real time. The detailed parameter formula is described as

$$
\Delta q_{\text {hip }}=k_{P}^{1}\left(\theta_{\text {torso }}^{d}-\theta_{\text {torso }}^{r}\right)+k_{D}^{1}\left(\dot{\theta}_{\text {torso }}^{d}-\dot{\theta}_{\text {torso }}^{r}\right),
$$

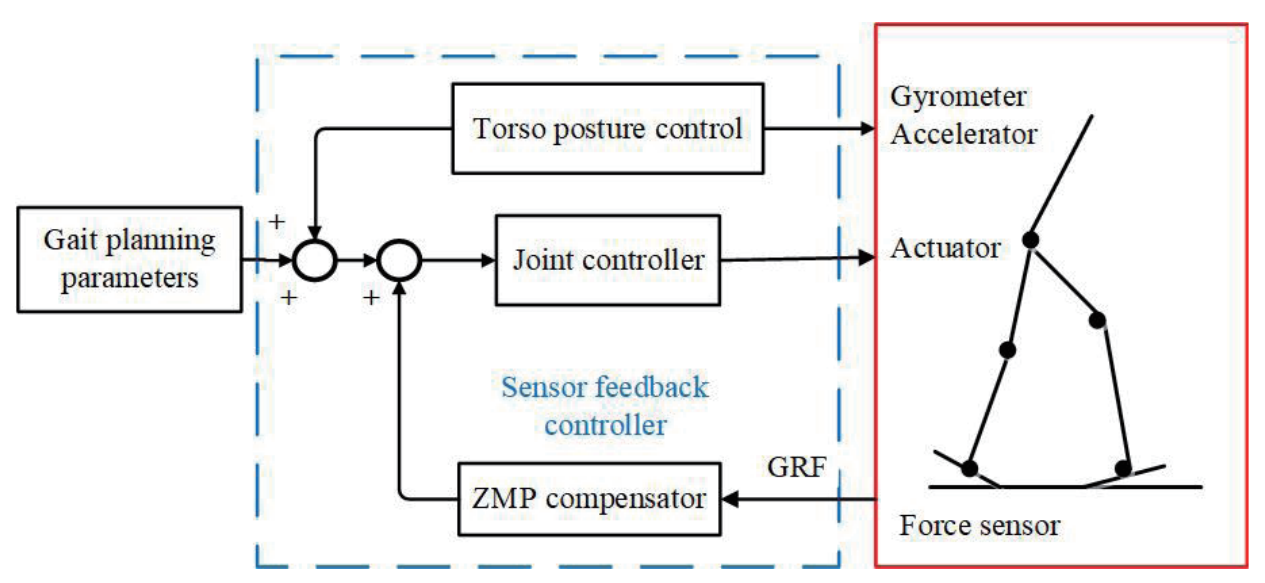

Fig. 3. (Color online) Sensory feedback controller. 
where $\theta_{\text {torso }}^{d}$ and $\dot{\theta}_{\text {torso }}^{d}$ represent the desired values of the torque angle, whereas $\theta_{\text {torso }}^{r}$ and $\dot{\theta}_{\text {torso }}^{r}$ represent the actual ones. $k_{p}^{1}$ and $k_{p}^{2}$ are modifying parameters.

\subsection{ZMP compensator}

The main goal of the ZMP compensator design lies in reducing the ZMP error between the actual and reference values, thus guaranteeing the stable walking in accordance with the preplanned trajectory. The ZMP compensator parameter is modified as

$$
\Delta q_{\text {ankle }}=k_{P}^{2}\left(x_{Z M P}^{d}-x_{Z M P}^{r}\right)+k_{D}^{2}\left(\dot{x}_{Z M P}^{d}-\dot{x}_{Z M P}^{r}\right)
$$

where $x_{Z M P}^{d}$ is the theoretical ZMP, which can be calculated using the planned gait, and $x_{Z M P}^{r}$ is the ZMP measured by force sensors.

\subsection{Parameter modification with policy gradient learning (PGL)}

In this subsection, the PGL algorithm is utilized to realize feedback parameter modification. This algorithm is a type of reinforced learning with the idea of "attempt-assessment-improvement". It is advantageous in solving the local optimal solution of policy, especially in the absence of a cost function situation.

Let $\boldsymbol{K}=\left[k_{P}^{1} k_{D}^{1} k_{P}^{2} k_{D}^{2}\right]$ be the feedback parameter set. Assume that the objective equation is differentiable for every parameter in it, then the optimal solution can be found by calculating the gradient of the objective equation $F(\boldsymbol{K})$,

$$
F(\boldsymbol{K})=\alpha\left(\left|\Delta q_{\text {hip }}\right|+\left|\Delta \bar{q}_{\text {hip }}\right|\right)+\beta\left(\left|\Delta q_{\text {ankle }}\right|+\left|\Delta \bar{q}_{\text {ankle }}\right|\right),
$$

where $\Delta \bar{q}_{\text {hip }}=\frac{1}{N} \sum_{i=1}^{N}\left(\theta_{\text {torso }}^{d}-\theta_{\text {torso }}^{r}\right), \Delta \bar{q}_{\text {hip }}=\frac{1}{N} \sum_{i=1}^{N}\left(\theta_{\text {torso }}^{d}-\theta_{\text {torso }}^{r}\right)$, and $N=\frac{t_{f}-t_{o}}{\Delta T}$ is the number of interpolation steps in the SSP. $\alpha$ and $\beta$ are the weighting factors.

Moreover, it is necessary to generate $p$ policies around $\boldsymbol{K}^{0} . \boldsymbol{K}_{m}^{i-1}(m=1,2, \ldots, p)$ stands for the policy set, where $p$ is proportional to the search space $s$. Thus, the policy set generation equation is described as

$$
\boldsymbol{K}_{m}^{i-1}=\boldsymbol{K}^{0}+\left[\rho_{1}, \rho_{2}, \ldots, \rho_{s}\right]
$$

where $\rho_{j} \in\left\{-\varepsilon_{j}, 0,+\varepsilon_{j}\right\}$.

In addition, the gradient $\nabla \boldsymbol{K}$ will be orthogonalized, and $\eta$ is the fixed step size factor. The pseudocode of the proposed PGL is as follows (Table 1). 
Table 1

Pseudocode of proposed PGL.

\begin{tabular}{|c|c|}
\hline & PGL algorithm \\
\hline & INPUT: $\boldsymbol{K}^{0}, \eta, N_{\text {iter }}$ \\
\hline & OUTPUT: $\boldsymbol{K}^{*}$ \\
\hline 1 & begin \\
\hline 2 & initialize $\boldsymbol{K} \leftarrow \boldsymbol{K}_{0}$ \\
\hline 3 & for each iteration $i=1$ to $N_{\text {iter }}$ \\
\hline 4 & generate $p$ random policies $\boldsymbol{K}_{m}^{i-1}$ near $\boldsymbol{K}^{i-1}$ \\
\hline 5 & evaluate $F(\boldsymbol{K})$ at all $p$ policies $\boldsymbol{K}_{m}^{i-1}$ \\
\hline 6 & for each parameter $j \in\{1,2 \ldots s\}$ \\
\hline 7 & Evaluate $\bar{F}_{-\varepsilon, j}, \bar{F}_{0}$ and $\bar{F}_{+\varepsilon, j}$ \\
\hline 8 & if $\bar{F}_{-\varepsilon, j}>\bar{F}_{0}$ and $\bar{F}_{+\varepsilon, j}>\bar{F}_{0}$ \\
\hline 9 & $\nabla \boldsymbol{K}_{j}^{i} \leftarrow 0$ \\
\hline 10 & else \\
\hline 11 & $\nabla \boldsymbol{K}_{j}^{i} \leftarrow \bar{F}_{+\varepsilon, j}-\bar{F}_{-\varepsilon, j}$ \\
\hline 12 & end if \\
\hline 13 & end for \\
\hline & $\nabla \boldsymbol{K}^{i}, \boldsymbol{K}^{i}$ \\
\hline 14 & $\sqrt{\left|\boldsymbol{K}^{i}\right|}$ \\
\hline 15 & $\boldsymbol{K}^{i} \leftarrow \boldsymbol{K}^{i-1}-\nabla \boldsymbol{K}^{i}$ \\
\hline 16 & end for \\
\hline 17 & return $\boldsymbol{K}^{*}$ \\
\hline 18 & end \\
\hline
\end{tabular}

Remark 4: By the real-time detection of robot walking data, the sensory feedback controller modifies the current posture of the robot in accordance with the planned gait, with the aim of reducing the errors between the measured and desired ZMPs. This is a sensor fusion concept, and the PGL can be used to solve the problem by iteration, realizing minimized cost function and model parameters.

\section{Simulation}

To verify the proposed methods given in Sects. 3 and 4, simulations are implemented. Table 2 lists the parameters of the robot used in the simulation. The sensor is mounted on the sole of the foot and obtains data to determine the ZMP during walking.

There are also some inequality constraints, including the friction coefficient, actuator motor torques, and GRFs. Let $|\tau|<250 \mathrm{~N} \cdot \mathrm{m}, \mu<0.4$, and the tangential component of GRF $\left|F_{\text {ext }}^{x}\right|<400 \mathrm{~N}$.

The ZMP positions along the $x$ - and $y$-directions are shown in Fig. 4 . It can be seen that the actual ZMP trajectory can track its reference trajectory. COM and ZMP positions are overlapped, which indicates that RL for parameter modification can improve the ZMP tracking accuracy, compared with the traditional PID control used in Ref. 28. 
Table 2

Biped robot parameters.

\begin{tabular}{lccc}
\hline & Torso & Thigh & Shank \\
\hline Link length $(\mathrm{m})$ & $L_{0}=0.788$ & $L_{1}=0.406$ & $L_{2}=0.473$ \\
Link mass $(\mathrm{kg})$ & $m_{0}=37.63$ & $m_{1}=5.53$ & $m_{2}=3.32$ \\
Link inertia $\left(\mathrm{kgm}^{2}\right)$ & $I_{0}=1.948$ & $I_{1}=0.076$ & $I_{2}=0.062$ \\
\hline
\end{tabular}

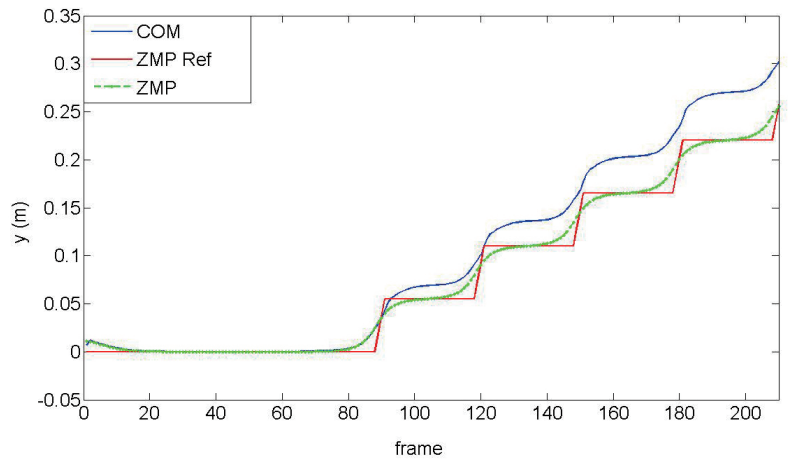

(a)

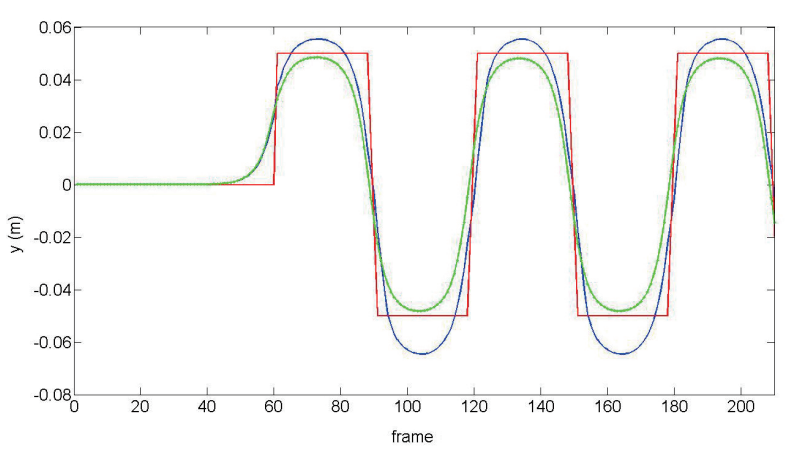

(b)

Fig. 4. (Color online) ZMP positions. (a) $X$ - and (b) $Y$-directions.
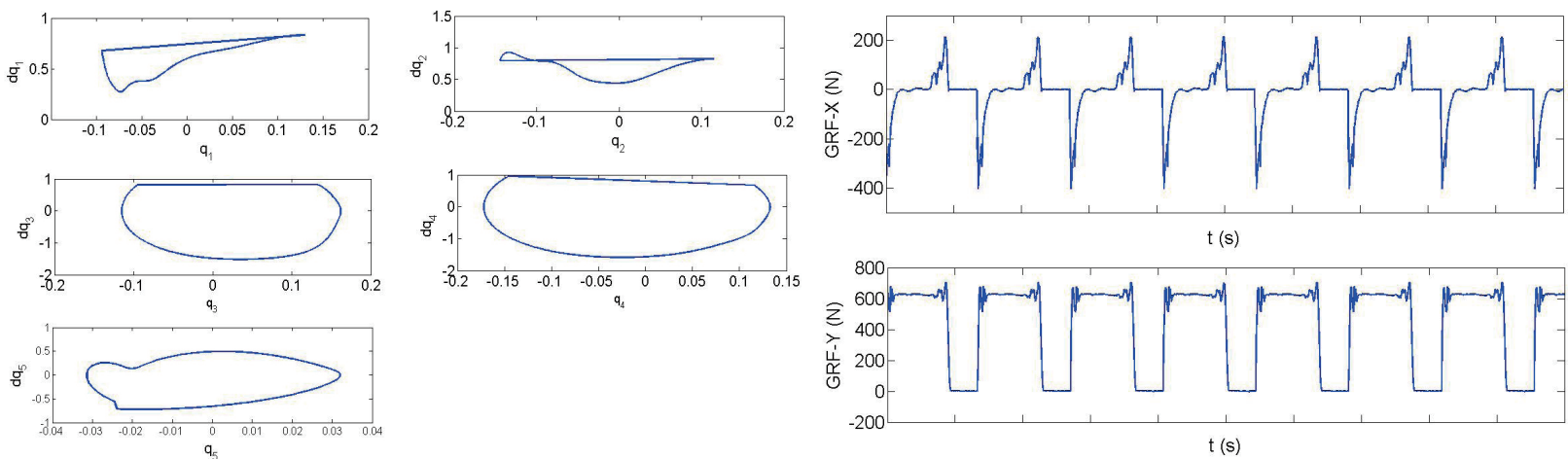

Fig. 5. (Color online) Phase plots of joint angles.

Fig. 6. (Color online) GRFs in $X$ - and $Y$-directions.

Figure 5 shows phase diagrams of joint angles. Each curve is in the form of a limit cycle, which indicates the walking process to be asymptotically stable and periodic. The GRFs along the $x$ - and $y$-directions are described in Fig. 6. The curves are periodic, representing the switch between the support leg and the swing leg. In the SSP, when the legs are off the ground, the GRFs are equal to zero. The tangential component of GRF also satisfies $\left|F_{\text {ext }}^{x}\right|<400 \mathrm{~N}$.

Figure 7 illustrates the hip and ankle angle rotation modifications with time, where the PGL algorithm described in Sect. 4.3 was utilized. The modifying policy is in real time. The red and blue curves describe the alternating continuous changes along with the left and right directions. The joint motor torques are described in Fig. 8 and satisfy the actor motor torque condition, $|\tau|<250 \mathrm{~N} \cdot \mathrm{m}$. It can be seen that all the actor motors are within the range of stable operation. 


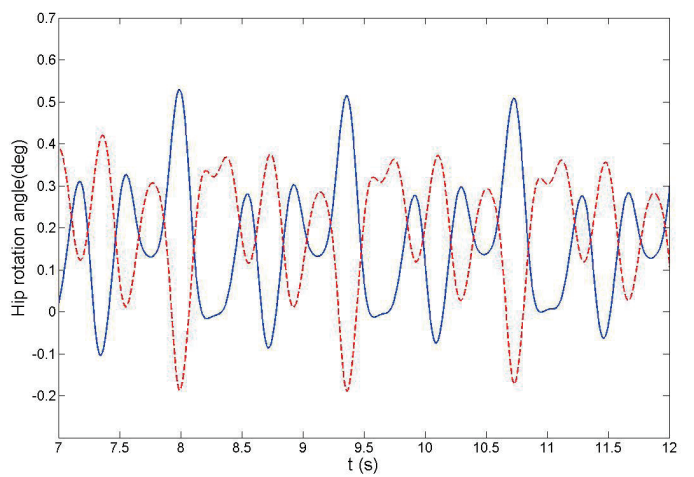

(a)

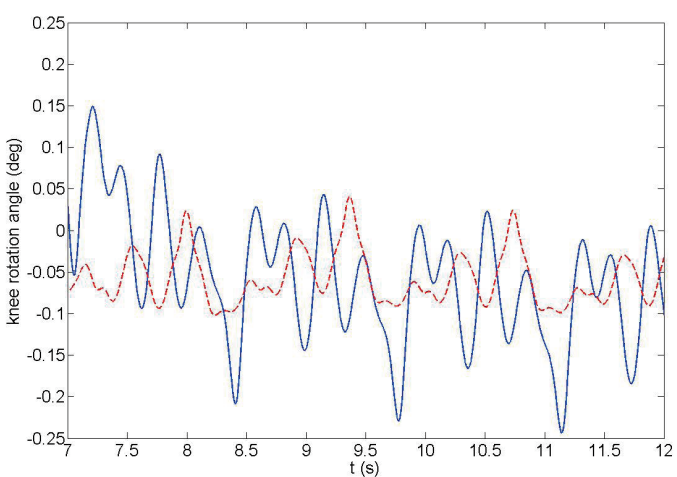

(b)

Fig. 7. (Color online) Rotation angle modifications with time. (a) Hip and (b) ankle.

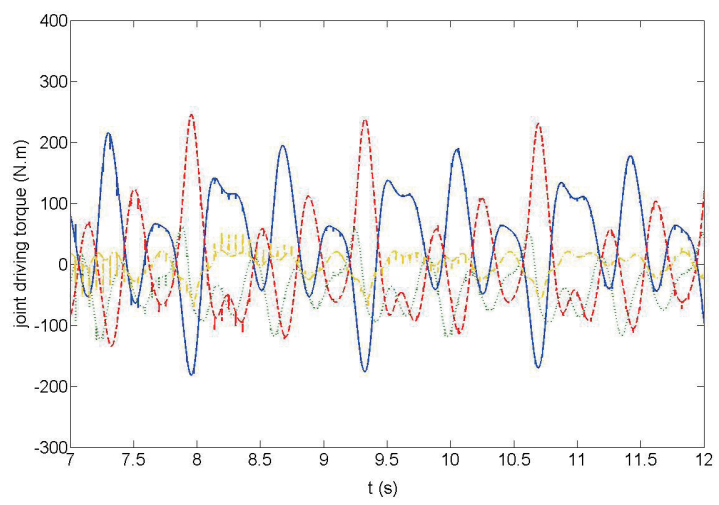

Fig. 8. (Color online) Joint motor torques with time.

\section{Conclusions}

In this paper, an overview of a smart city was discussed, including increasingly rapid development in various countries, the core technologies of sensors, and their application scenarios.

As one of the many applications in smart cities, robotic sensor systems are investigated. They can integrate motion, interaction, and manipulation functions to assist or replace human beings in daily tasks. A gait planning strategy was proposed for a 3D biped walker with kinetic constraints and the ZMP stability criterion. The walking parameters were regulated via sensory feedback control. The sensory controller consisted of the torso posture controller, ZMP compensator, and impact reducer; the parameters of these controllers are automatically regulated in each walking cycle by the policy gradient RL method. The proposed method provides an optimal walking gait and improves the trajectory tracking accuracy. In the future, a robust sensory controller against external disturbance for the robotic sensor applications is expected. In addition, human beings tend to utilize learned experiences to realize more complicated movements in high-dimensional environments in smart cities. 


\section{Acknowledgments}

This work is supported by National Natural Science Foundation of China under Grant (61573260, 61773289, U1713211), Science and Technology Commission of Shanghai Municipality under Grant (16JC1401200), Shanghai International Science and Technology Cooperation Project (18510711100), Shanghai Shuguang Project (18SG18), Shanghai Natural Science Foundation (17ZR1445800), and Fundamental Research Funds for the Central Universities.

\section{References}

1 S. Alawadhi, A. Aldama-Nalda, H. Chourabi, J. R. Gil-Garcia, S. Leung, and S. Mellouli: Lect. Notes Comput. Sci. 7443 (2012) 40.

2 R. Carli, M. Dotoli, R. Pellegrino, and L. Ranieri: Proc. 2013 IEEE Systems, Man, and Cybernetics (IEEE, 2013) 1288-1293.

3 V. Gungor, B. Lu, and G. Hancke: IEEE Trans. Ind. Electron. 57 (2010) 3557.

4 G. P. Hancke: J. Sens. 13 (2012) 393.

5 J. A. Khan, Y. Ghamridoudane, and D. Botvich: IEEE Trans. Veh. Technol. 65 (2016) 9529.

6 Y. C. Wang and G. W. Chen: IEEE Trans. Veh. Technol. 99 (2017) 1.

7 B. Tang, C. Zhen, G. Hefferman, S. Y. Pei, T. Wei, H. B. He, and Q. Yang: IEEE Trans. Ind. Inf. 13 (2017) 2140.

8 H. Zhang, X. Zhou, Z. P. Wang, H. C. Yan, and J. Sun: IEEE Trans. Cybern. 99 (2018) 1.

9 Z. P. Wang, G. B. Li, X. Z. Chen, H. Zhang, and Q. J. Chen: IEEE Trans. Ind. Electron. 66 (2019) 5343.

10 M. B. A. Haghighat, A. Aghagolzadeh, and H. Seyedarabi: Comput. Electr. Eng. 37 (2011) 789.

11 D. Zhang and Y. C. Kiat: IEEE Trans. Parallel Distrib. Syst. 24 (2013) 479.

12 H. Zhang, Z. P. Wang, H. C. Yan, F. W. Yang, and X. Zhou: IEEE Trans. Cybern. 10 (2018) 1.

13 R. H. Yang, H. Zhang, G. Feng, H. C. Yan, and Z. P. Wang: Autom. 102 (2019) 129.

14 D. Gouaillier, C. Collette, and C. Kilner: Proc. 2010 IEEE-RAS Int. Conf. Humanoid Robots (IEEE, 2010) $448-454$

15 G. M. Atmeh, I. Ranatunga, D. O. Popa, K. Subbarao, F. Lewis, and P. Rowe: Proc. 2014 American Control (IEEE, 2014) 2887-2892.

16 D. Katić and M. Vukobratović: J. Intell. Rob. Syst. 37 (2003) 117.

17 I. Hashlamon and K. Erbatur: Proc. 2013 IEEE Int. Conf. Mechatronics (IEEE, 2013) 248-253.

18 H. Iyad, M. M. Gülhan, A. Orhan, and K. Erbatur: Robotica 35 (2017) 21.

19 M. Shimojo, T. Araki, A. Ming, and M. Ishikawa: Proc. 2006 IEEE Int. Conf. Robotics and Automation (IEEE, 2006) 1200-1205.

20 H. Rodolphe and B. Espiau: IEEE Trans. Rob. 24 (2008) 191.

21 H. C. Lin, K. P. Chen, B. R. He, K. C. Hsiao, and H. C. Zo: Sens. Mater. 29 (2017) 757.

22 J. P. Yu, W. Wang, X. Li, and Z. Z. Zhou: Sens. Mater. 29 (2017) 905.

23 S. Kajita, F. Kanehiro, K. Kaneko, K. Fujiwara, K. Yokoi, and H. Hirukawa: Proc. 2006 IEEE/RSJ Int. Conf. Intelligent Robots and Systems (IEEE, 2006) 2993-2999.

24 C. Zhu, Y. Tomizawa, X. Luo, and A. Kawamura: Proc. 2004 IEEE Int. Conf. Robotics and Biomimetics (IEEE, 2004) 425-430.

25 B. J. Lee, D. Stonier, Y. D. Kim, J. K. Yoo, and J. H. Kim: IEEE Trans. Robot. 24 (2008) 917.

26 O. Ayhan and K. Erbatur: Proc. 30th Ann. Conf. IEEE Industrial Electronics Society (IEEE, 2004) 1797-1802.

27 J. Wallen, S. Gunnarsson, R. Henriksson, S. Moberg, and M. Norrlof: Proc. 2009 IEEE Conf. Decision and Control (IEEE, 2009) 458-463.

28 B. Aghbali, A. Yousefi-Koma, A. G. Toudeshki, and A. Shahrokhshahi: Proc. 2013 First RSI/ISM Int. Conf. Robotics and Mechatronics (IEEE, 2013) 530-534. 


\section{About the Authors}

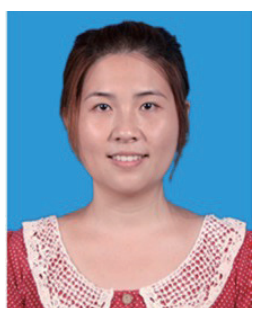

Helin Wang received her B.S. degree in electric engineering and automation from Jiangsu Normal University, China, in 2012, and her M.S. degree in automatic control from Shanghai Electric Power University, Shanghai, China, in 2015. She is currently working toward a Ph.D. degree in control theory and control engineering at Tongji University, Shanghai, China. Her current research interests include intelligent control of robotic systems and sensors. (wh17228@tongji.edu.cn)

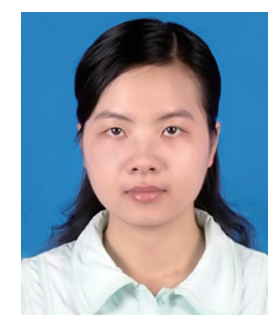

Hao Zhang received her B.S. degree in automatic control from the Wuhan University of Technology, Wuhan, China, in 2001 and her Ph.D. degree in control theory and control engineering from the Huazhong University of Science and Technology, Wuhan, China, in 2007. She is currently a professor at Tongji University, Shanghai, China. Her research interests include networkbased control systems, multiagent systems, and complex networks. (zhang_ hao@tongji.edu.cn)

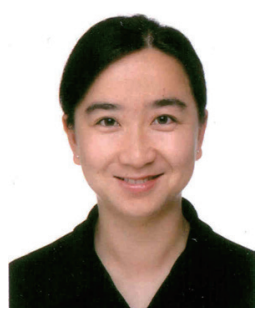

Zhuping Wang received her B. Eng. and M. Eng. degrees from Northwestern Polytechnic University, China, in 1994 and 1997, respectively, and her Ph.D. degree in automatic control from the National University of Singapore, Singapore, in 2003. She is currently a professor at Tongji University, Shanghai, China. Her research interests include intelligent control of robotic systems and nonholonomic control systems. (elewzp@tongji.edu.cn)

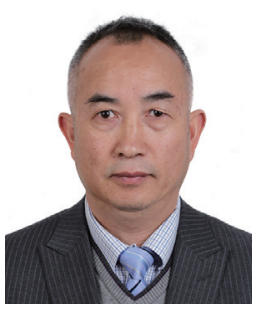

Qijun Chen received his B.S. degree in automatic control from the Huazhong University of Science and Technology, China, in 1987, his M.S. degree in control engineering from Xi'an Jiaotong University, Xi'an, China, in 1990, and his Ph.D. degree in control theory and control engineering from Tongji University, Shanghai, China, in 1999. He is currently a professor at Tongji University, Shanghai, China. His current research interests include networkbased control systems and robotics. (qjchen@tongji.edu.cn) 